\section{Jacques Drouin}

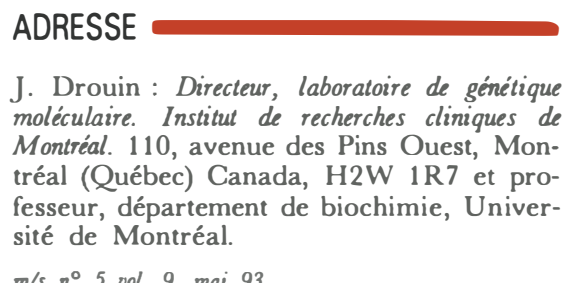

\title{
Répression transcriptionnelle : glucocorticoïdes et pro-opiomélanocortine
}

Les glucocorticoïdes peuvent activer ou inhiber la transcription de nombreux gènes. Les mécanismes de l'activation commencent à être bien connus ; ils comportent la fixation sur l'élément de réponse, une séquence palindromique d'ADN, d'un dimère du complexe entre l'hormone et le récepteur. En revanche, les mécanismes des actions négatives des glucocorticoïdes sont plus mal connus et sont d'ailleurs divers. La régulation négative de la transcription du gène de la pro-opiomélanocortine (POMC, précurseur de l'ACTH) met en jeu un élément de réponse négatif dont la particularité semble être de fixer un trimère de récepteur, deux molécules étant fixées sur une face de l'ADN et une troisième sur l'autre face. Cette configuration particulière pourrait interférer avec le processus d'activation normal du complexe d'initiation de la transcription par des activateurs situés plus à distance.

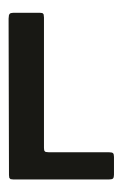

es hormones constituent avec le système nerveux les médiateurs de l'échange d'information entre les tissus des organismes supérieurs. Elles influencent ainsi la majorité des processus physiologiques et leurs actions s'exercent à plusieurs niveaux sur la cellule cible. Au cours du développement, les hormones sont des signaux importants d'induction de la différenciation cellulaire. Plusieurs de ces actions résultent de la régulation de la transcription de gènes cibles dans les cellules qui expriment les récepteurs hormonaux appropriés. Les récepteurs des hormones stéroïdes sont à la fois sites de liaison du ligand hormonal et facteurs de transcription. Il a donc été beaucoup plus facile de passer de l'action des hormones au contrôle de la transcription dans ces systèmes qu'il ne l'a été pour les hormones qui agissent par l'intermédiaire d'un récepteur membranaire et d'une cascade de signaux intracellulaires. C'est ainsi que le récepteur des glucocorticoïdes (RG) fût le premier facteur de transcription de mammifere cloné [1] et 


\section{RÉFÉRENCES}

1. Hollenberg SM, Weinberger C, Ong ES, Cerelli G, Oro A, Lebo R, Thompson EB, Rosenfeld MG, Evans RM. Primary structure and expression of a functional human glucocorticoid receptor cDNA. Nature 1985 ; $318: 635-41$

2. Drouin, J. Repression of transcription by nuclear receptors. In : Frontiers in molecular biology : Steroid hormone action, edited by Parker, M.G. England: Oxford University Press, 1993, p. in press.

3. Beato $M$. Gene regulation by steroid hormones. Cell 1989 ; 56 : 335-44.

4. Evans RM. The steroid and thyroid hormone receptor superfamily. Science 1988 ; $240: 889-95$

5. Sanchez ER, Meshinchi S, Tienrungroj W, Schlesinger J, Toft DO, Pratt WB. Relationship of the $90-\mathrm{kDa}$ murine heat shock protein to the untransformed and transformed states of the $\mathrm{L}$ cell glucocorticoid receptor. I Biol Chem 1987; 262 : 6986-91

6. Drouin J, Sun YL, Tremblay S, Lavender P, Schmidt TJ, De Léan A, Nemer $M$. Homodimer formation is rate-limiting for high affinity DNA binding by glucocorticoid receptor. Mol Endocrinol 1992 ; 6 : 1299-309.

7. Frankel AD, Kim PS. Modular structure of transcripiton factors : implications for gene regulation. Cell 1991; 65: 717-9.

8. Shemshedini L, Knauthe R, SassoneCorsi P, Pornon A, Gronemeyer H. Cellspecific inhibitory and stimulatory effects of fos and jun on transcription activation by nuclear receptors. EMBO J 1991; 10 : 3839-49.

9. Yang-Yen HS, Chambard JC, Sun YL, Smeal T, Schmidt TJ, Drouin J, Karin M. Transcriptional interference between c-jun and glucocorticoid receptor due to mutual inhibition of DNA-binding activity. Cell $1990 ; 62$ : 1205-15.

10. Baniahmad A, Steiner C, Kîhne AC, Renkawitz R. Modular structure of a chicken lysozyme silencer : involvement of an unusual thyroid hormone receptor binding site. Cell 1990 ; 61 : 505-14.

11. Baniahmad A, Kîhne AC, Renkawitz R. A transferable silencing domain is present in the thyroid hormone receptor, in the $v$-erbA oncogene product and in the retinoic acid receptor. EMBO J 1992 ; 11 : 1015-23.

12. Bodenner DL, Mroczynski MA, Weintraub BD, Radovick S, Wondisford FE. A detailed functional and structural analysis of a major thyroid hormone inhibitory element in the human thyrotropin beta-subunit gene. $J$ Biol Chem 1991; 266 : 21666-73.

13. Nããr AM, Boutin J-M, Lipkin SM, Yu VC, Holloway JM, Glass CK, Rosenfeld MG. The orientation and spacing of core DNA-binding motifs dictate selective transcriptional responses to three nuclear recep- que plusieurs concepts généraux du contrôle de la transcription sont dus à l'étude de ce facteur de transcription. Les progrès ont été d'autant plus spectaculaires et rapides que les récepteurs des hormones stéroïdes font partie d'une très grande famille de facteurs de transcription, les récepteurs nucléaires, dont l'activité est contrôlée par des ligands qui incluent non seulement les hormones stéroïdes mais aussi les hormones thyroïdiennes, la vitamine $\mathrm{D}$ et des morphogènes comme l'acide rétinoïque. Les récepteurs nucléaires, que ce soit ceux des hormones stéroïdes ou les autres, répriment probablement autant de gènes cibles qu'ils n'en stimulent. Toutefois, peu d'études ont porté sur le mécanisme de la répression transcriptionnelle due aux récepteurs nucléaires, et par conséquent ces mécanismes demeurent encore mal définis [2]. Les mécanismes proposés pour expliquer l'action transcriptionnelle négative des récepteurs nucléaires seront discutés dans cet article. Une attention particulière sera portée au mécanisme de la répression transcriptionnelle du gène de la pro-

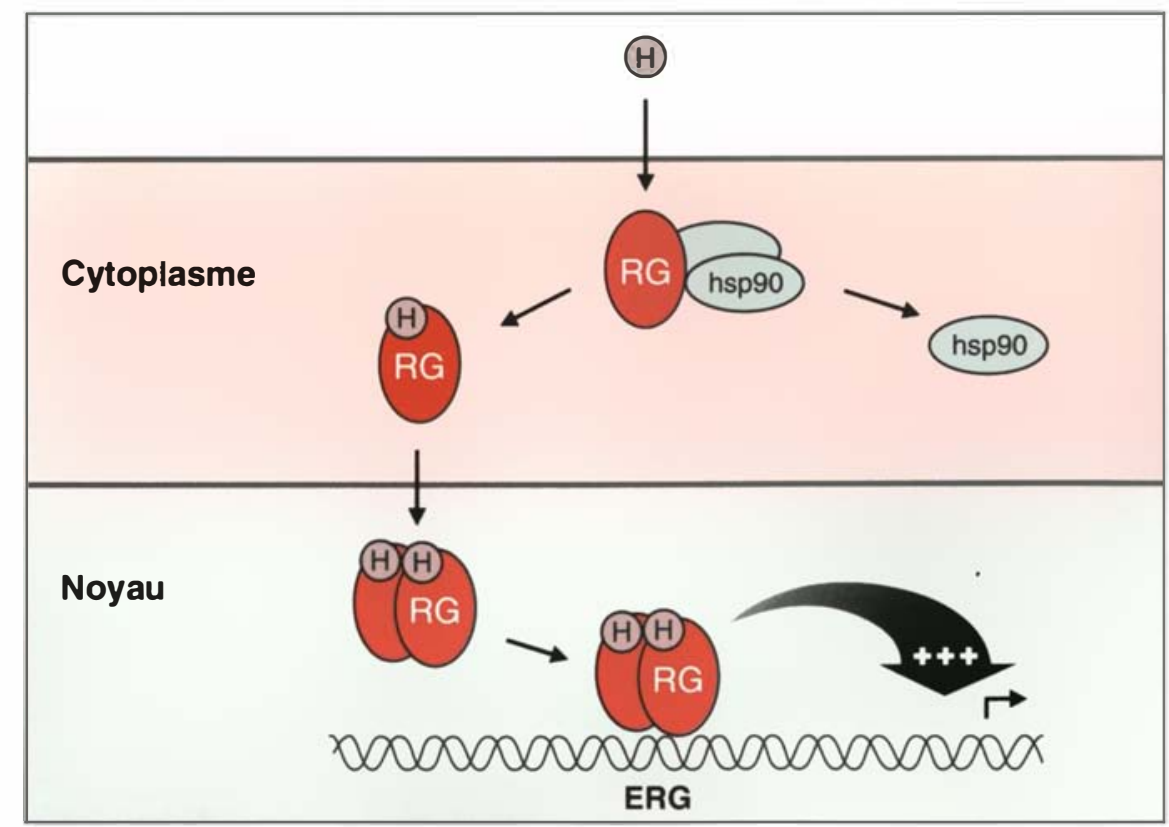

Figure 1. Activation transcriptionnelle induite par le récepteur des glucocorticoïdes (RG) et par son ligand hormonal, les glucocorticoïdes (H). En absence de l'hormone, le récepteur se trouve principalement dans le cytoplasme sous forme d'un complexe avec la protéine du choc thermique hsp90. La liaison de l'hormone induit la dissociation de ce complexe, la dimérisation du récepteur et sa translocation au noyau où il peut agir sur ses cibles géniques, les éléments de réponse aux glucocorticoïdes (ERG). opiomélanocortine (POMC) par les glucocorticoïdes et leurs récepteurs.

\section{Activation transcriptionnelle par les récepteurs nucléaires}

Les récepteurs nucléaires sont des facteurs de transcription dont l'activité transcriptionnelle est conditionnelle à la liaison du ligand $[3,4]$. Ainsi, le récepteur des glucocorticoïdes (RG), mais aussi celui des œstrogènes (RE), de la progestérone (RP), des androgènes (RA), des minéralocorticoïdes (RM) et plusieurs autres dont le ligand n'est pas connu, sont constitués de domaines modulaires et interpour la liaison à l'ADN et pour la transactivation. Dans certains cas, comme pour le RG, ces récepteurs sont présents dans le cytoplasme en l'absence d'hormone et le premier effet de l'hormone est de provoquer la dissociation du récepteur de son complexe cytoplasmique qui contient quelques protéines dont celle du choc thermique hsp90 [5] (figure 1). Après changeables pour la liaison du ligand, 
son activation, le $\mathrm{RG}$ migre vers le noyau et forme des homodimères. Ceux-ci lient avec une haute affinité les éléments de réponse aux glucocorticoïdes (ERG) présents dans les promoteurs des gènes cibles des glucocorticoïdes [3, 6]. Ces ERG sont constitués d'une séquence d'ADN de 15 paires de base qui est très conservée dans différents gènes cibles ainsi que dans différentes espèces [3]. Ils suffisent pour conférer la réponse hormonale et leur effet peut se manifester indépendamment de leur position ou leur orientation dans le promoteur. Leur séquence est constituée de deux hexanucléotides inversés et séparés par trois paires de base. Dès sa liaison à l'ADN, le RG active très rapidement la transcription et cette action est relayée par différents domaines de transactivation présents dans le récepteur [7]. Il semble que plusieurs réccpteurs contiennent plus d'un domaine de transactivation et que selon le type cellulaire et le contexte du promoteur auquel s'associc le récepteur, l'un et/ou l'autre de ces domaines de transactivation participe à l'activation transcriptionnelle [8]. En résumé, l'activation de la transcription par les récepteurs nucléaires est relativement simple, bien qu'il y ait des différences d'un récepteur à l'autre, et l'on pourrait tout simplement conclure que ceux-ci sont des facteurs transcriptionnels comme d'autres, avec la particularité d'une activité dépendante de la liaison avec le ligand.

\section{Répression transcriptionnelle par les récepteurs nucléaires}

Bien qu'il soit connu depuis fort longtemps que les stéroïdes agissent sur leur tissu cible en réprimant presque autant de gènes qu'ils en stimulent, nous comprenons encore mal le ou les mécanismes par lesquels leurs récepteurs répriment la transcription. En effet, il est encore difficile d'identifier un mécanisme général par lequel la répression transcriptionnelle s'exercerait et il semblerait plutôt que différents mécanismes soient responsables de la répression de différents gènes cibles. Ainsi, il n'est pas possible de dégager une séquence con-

En absence du ligand (H)

En présence du ligand $(H)$
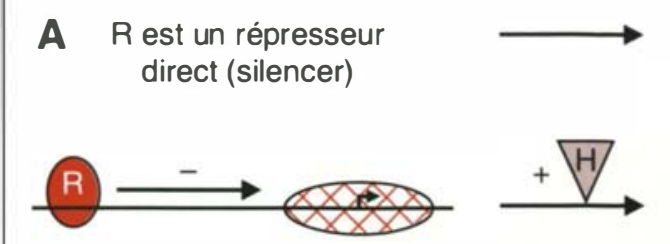

Activation
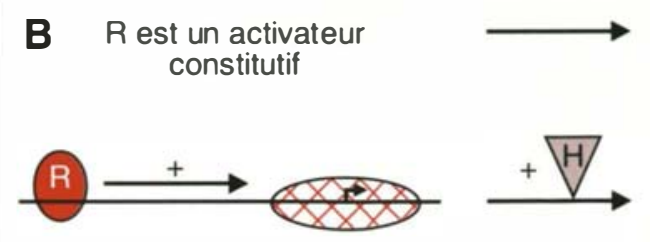

Répression
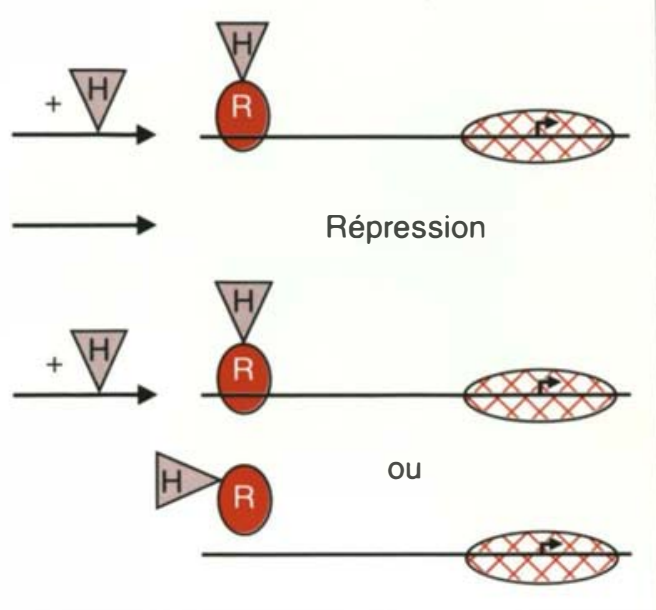

Figure 2. Le récepteur des hormones thyroïdiennes (RT) semble différer des autres récepteurs comme le RG par son activité transcriptionnelle même en l'absence de son ligand. Lorsque le récepteur est lié à un élément positif (A), le RT réprimerait la transcription de base en absence du ligand et la présence de l'hormone lèverait cette inhibition. Inversement, lorsqu'il est lié à un élément négatif (B), ce récepteur activerait la transcription de base et cette activité serait inhibée par la liaison du ligand hormonal.

sensus d'ADN qui représenterait un ERG négatif (ERGn) et il en est de même pour les gènes qui sont réprimés par d'autres récepteurs nucléaires. Il semble même que dans certains cas, la répression induite par un récepteur nucléaire ne requiert pas une interaction directe de ce dernier avec l'ADN et de fait, ce serait plutôt par des interactions entre protéines que le récepteur inhiberait la transcription [9]. Afin de refléter la diversité des mécanismes de la répression transcriptionnelle, quelques exemples impliquant des récepteurs nucléaires seront brièvement discutés.

1. Le cas du récepteur des hormones thyroïdiennes

Les récepteurs des hormones thyroïdiennes (RT) semblent être différents des autres récepteurs, tels $R G, R P$, RM et RE parce qu'ils sont actifs au niveau de la transcription même en l'absence du ligand (figure 2). Dans ce cas, la liaison avec le ligand semble renverser l'effet transcriptionnel du récepteur. Ainsi, en ce qui concerne les gènes dont la transcription est stimulée par les hormones thyroïdiennes (figure $2 A$ ), le récepteur réprime la transcription constitutive en l'absence $\mathrm{du}$ ligand et le ligand renverse cet effet [10,11] ; inversement, les gènes dont la transcription est répriméc par l'hormone thyroïdienne (figure 2B) comme le gène encodant la sous-unité $\beta$ de l'hormone thyréotrope (TSH), sont activés de façon constitutive par le récepteur et l'action de l'hormone consiste à supprimer cet effet [12, 13]. Il apparaît donc que le mécanisme de l'action transcriptionnclle du récepteur de l'hormone thyroïdienne (et peut-être aussi certains autres récepteurs qui peuvent former des hétérodimères avec celui-ci, comme le récepteur de l'acide rétinoïque, $\mathrm{RAR}$, et le récepteur de l'acide 9-cis rétinoïque, $\mathrm{RXR}$ ) soit différent de celui des récepteurs qui semblent toujours agir sous forme d'homodimère tel que le $R G$, le $R E$ et le $R P$.

\section{Répression dépendant d'une liai-} son récepteur-ADN

Sites de liaison inertes. Puisque les récepteurs nucléaires sont de puissants activatcurs de la transcription lorqu'ils agissent sur les "éléments de 


\section{RÉFÉRENCES}

14. Glass CK, Holloway JM, Devary OV, Rosenfeld MG. The thyroid hormone receptor binds with opposite transcriptional effects to a common sequence motif in thyroid hormone and estrogen response elements. Cell $1988 ; 54: 313-23$

15. Keleher CA, Goutte C, Johnson AD The yeast cell-type-specific repressor alpha2 acts cooperatively with a non-cell-typespecific protein. Cell 1988 ; 53 : 927-36.

16. Diamond MI, Miner JN, Yoshinaga SK, Yamamoto KR. Transcription factor interactions : selectors of positive or negative regulation from a single DNA element. Science $1990 ; 249$ : 1266-72.

17. O'Halloran TV, Frants B, Shin MK, Ralston DM, Wright JG. The merR heavy metal receptor mediates positive activation in a topologically novel transcription complex. Cell 1989 ; 56 : 119-29.

18. Gill G, Ptashne M. Negative effect of the transcriptional activator GAL4. Nature 1988 ; 334: 721-4.

19. Schüle $R$, Rangarajan $P$, Kliewer $S$, Ransone LJ, Bolado J, Yang N, Verma IM, Evans RM. Functional antagonism between oncoprotein c-jun and the glucocorticoid receptor. Cell $1990 ; 62$ : 1217-26.

20. Jonat C, Rahmsdorf HJ, Park K-K, Cato ACB, Gebel S, Ponta H, Herrlich P. Antitumor promotion and antiinflammation : down-modulation of AP-1 (fos/jun) activity by glucocorticoid hormone. Cell $1990 ; 62$ : 1189-204.

21. Lucibello FC, Slater EP, Jooss KU, Beato M, Müller R. Mutual transrepression of fos and the glucocorticoid receptor : involvement of a functional domain in fos which is absent in fos $\mathrm{B} . E M B O J 1990 ; 9$ : 2827-34.

22. Gagner J-P, Drouin J. Tissue-specific regulation of pituitary pro-opiomelanocortin gene transcription by corticotropin-releasing hormone, 3', 5'-cyclic adenosine monophosphate, and glucocorticoids. Mol Endocrinol $1987 ; 1: 677-82$

23. Drouin J, Sun YL, Nemer M. Regulatory elements of the pro-opiomelanocortin gene. Pituitary specificity and glucocorticoid repression. Trends in Endocrinol Metab 1990 ; 1 : 219-25.

24. Drouin J, Trifiro MA, Plante RK, Nemer M, Eriksson P, Wrange Ö. Glucocorticoid receptor binding to a specific DNA sequence is required for hormone-dependent repression of pro-opiomelanocortin gene transcription. Mol Cell Biol $1989 ; 9$ : 5305-14.

25. Drouin J, Sun YL, Chamberland M, Gauthier Y, De Léan A, Nemer M, Schmidt TJ. Novel glucocorticoid receptor complex with DNA element of the hormonerepressed POMC gene. EMBO J 1993 ; 12 : 145-56.

26. Therrien $\mathrm{M}$, Drouin J. Pituitary POMC expression requires synergistic interactions of several regulatory elements. $\mathrm{Mol}$ réponse hormonale " (ERH), l'action de ces mêmes récepteurs comme répresseurs transcriptionnels exige, dans la plupart des cas, que leur fonction de transactivation soit silencieuse dans le contexte des gènes réprimés. Ceci pourrait être accompli par l'interaction du récepteur avec une séquence d'ADN qui lie ce dernier efficacement mais qui, à l'opposé des ERH, ne permet pas des changements de conformation nécessaires à la fonction de transactivation. Un tel "site de liaison inerte " représenterait à proprement parler un ERH négatif puisqu'il aurait la seule propriété de conférer une régulation négative. L'ERGn identifié dans le promoteur proximal du gène de la POMC semble être un exemple d'un tel site inerte (décrit plus bas). D'autres ERH, comme l'élément de réponse aux œstrogènes (ERE), pourraient se comporter comme un élément positif pour un récepteur (RE, dans ce cas) et comme un élément inerte pour un autre récepteur (le RT, dans ce cas) [14].

Compétition. La compétition entre deux facteurs pour la liaison de séquences d'ADN qui se chevauchent est le mécanisme classique de la répression (figure 3). C'est le mécanisme de répression utilisé par plusieurs gènes bactériens comme l'opéron lactose décrit il y a bien longtemps par Monod et ses collaborateurs. On a proposé ce modèle pour expliquer la répression par plusieurs récepteurs nucléaires mais les travaux les plus récents suggèrent que, dans la plupart des cas, ce mécanisme ne soit pas le bon.

Interférence. La répression par interférence résulte de la liaison du répresseur à un site sur l'ADN qui ne lie aucune autre protéine (figure 3). A l'opposé d'un élément silencer, la liaison du répresseur n'est pas suffisante pour relayer la répression. Le contexte du promoteur est essentiel au mécanisme par lequel le répresseur inhibe la transcription. Par exemple, la liaison du répresseur pourrait empêcher des interactions entre des activateurs liés en amont du site de liaison du répresseur et la machinerie de base de transcription. Cooccupation (quenching). Un autre mécanisme de répression résulte de la liaison d'un activateur et d'un répres- seur à des sites contigus. L'interaction entre les deux protéines liées à l'ADN produit une atténuation de la fonction transactivatrice de l'activateưr (figure 3). Ce mécanisme semble responsable de la répression produite par la protéine $\alpha 2$ de la levure sur des gènes activés par le facteur de transcription MCM1 [15]. Dans ce cas, il a été montré que les deux protéines lient de façon coopérative leurs sites contigus, et que la protéine $\alpha 2$ empêche la transactivation par MCM1. Un mécanisme similaire a été invoqué pour expliquer la répression du gène de la proliférine par les glucocorticoïdes [16]. Dans ce cas, un site de faible affinité pour le RG se trouve juxtaposé à un site AP1 qui peut lier les homodimères du protooncogène $c-j u n$ ou les hétérodimères constitués de ce même protooncogène et de c-fos. Selon la nature du complexe AP1 (jun/jun ou jun/fos), il semblerait que le récepteur des glucocorticoïdes produise, soit une activation transcriptionnelle synergique, soit un effet répresseur. De tels éléments de régulation sont dits composites.

Répresseur direct ou silencer. Ce mécanisme de répression est l'opposé du mécanisme d'activation par l'ERG positif. En effet, les éléments agissant par ce mécanisme sont en eux-mêmes suffisants pour conférer une réponse transcriptionnelle négative quelque soit leur orientation ou leur position dans le promoteur (figure 3). Ainsi, l'action d'un répresseur direct ou silencer requiert uniquement la liaison à l'ADN de cette protéine et son effet s'exerce directement sur la machinerie de transcription. La protéine régulatrice bactérienne mer $R$ réprime la transcription en se liant à l'ADN de l'opérateur avec une affinité très élevéc : à cause de contraintes exercées sur la conformation de l'ADN, cette interaction empêche la formation de "complexe ouvert " [17]. Lorsque le mercure lie la protéine $m e r R$, l'interaction de cette protéine avec l'ADN se relâche et mer $R$ agit maintenant comme un activateur de la transcription. En ce qui concerne les récepteurs nucléaires, le meilleur exemple d'un récepteur qui agit comme un silencer serait le RT en l'absence de son ligand [11]. Il en est de même du mutant oncogénique du 
A • Répression dépendant d'une liaison récepteur(R)-ADN

Compétition

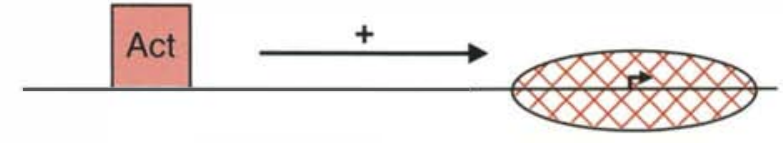

Interférence

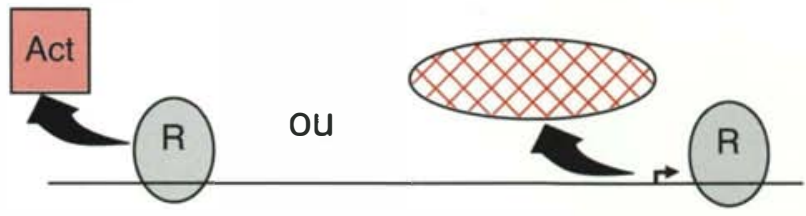

Co-occupation

(quenching)
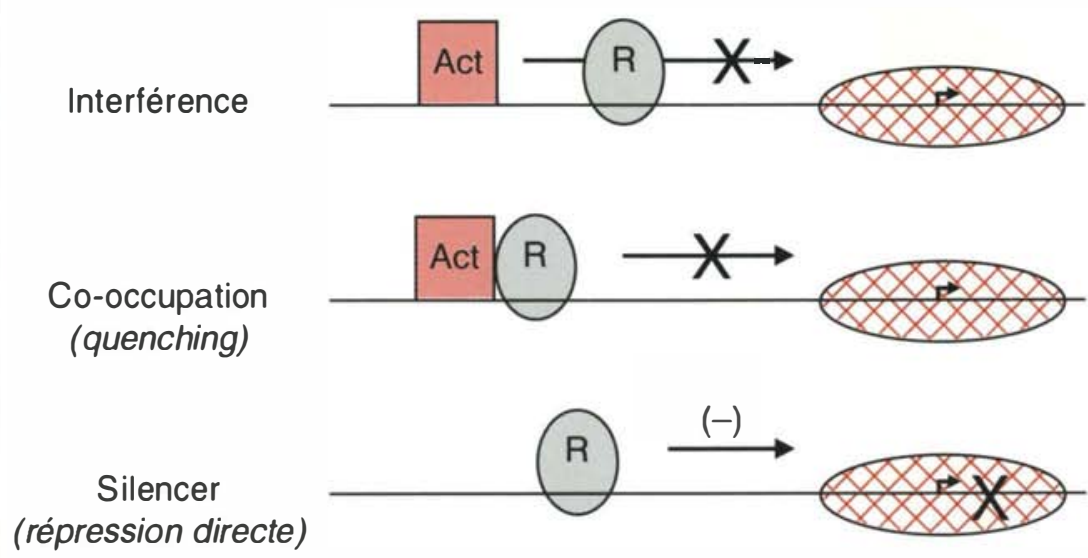

\section{B • Répression sans interaction récepteur(R)-ADN}

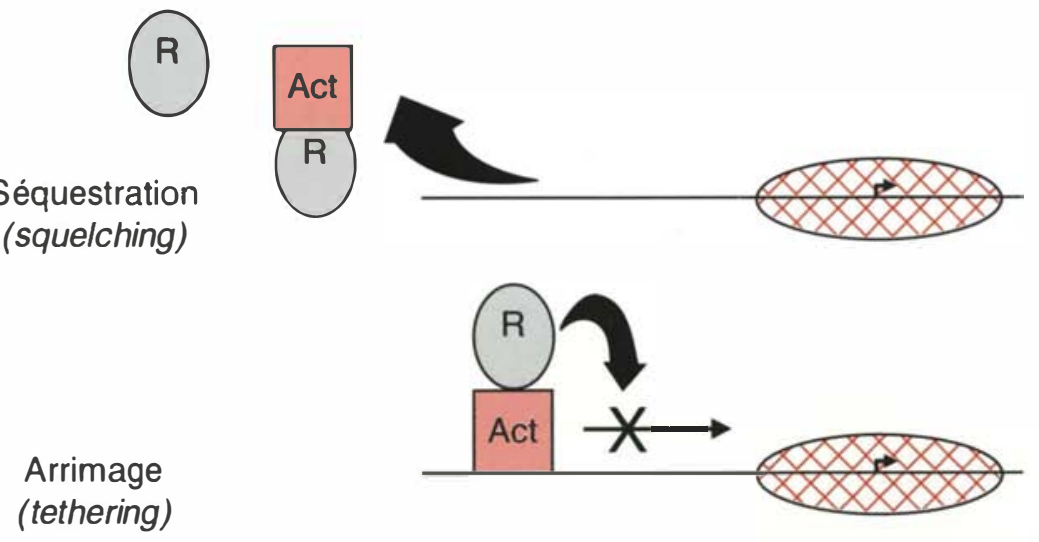

Figure 3. Différents mécanismes par lesquels un répresseur peut inhiber la transcription. A) Les mécanismes qui dépendent de la liaison à l'ADN du répresseur sont ceux dits de compétition, d'interférence, de co-occupation ou de répression directe (silenceur). B) Deux mécanismes ont été récemment proposés pour expliquer la répression transcriptionnelle qui ne dépend pas de la liaison directe du répresseur à l'ADN, soit les mécanismes de séquestration et d'arrimage. Ces mécanismes sont illustrés sur un promoteur type où sont représentés la machinerie de transcription de base représentée par une ellipse entourant le site d'initiation de la transcription, le site de liaison d'un facteur de transcription activateur (Act), et dans les cas appropriés, un site de liaison du répresseur $(R)$.

$m / s n^{\circ} 5$ vol. 9, mai 93
$\mathrm{RT}$, le produit de l'oncogène viral verb $A$, qui n'est pas sensible au ligand naturel, l'hormone thyroïdienne.

3. Répression indépendante d'une liaison récepteur-ADN

Séquestration (squelching). La séquestration ou squelching de facteurs de transcription a d'abord été décrite comme un artefact des expériences de transfert de gènes dans lesquelles un activateur était surexprimé [18]. Ainsi, la surexpression d'un activateur produit la répression d'un gène cible pour un second activateur; le premier activateur ne lie pas la même séquence que le second, et de plus, le domaine de liaison à l'ADN du premier activateur n'est pas requis pour cet effet. Les deux protéines interagissent entre elles indépendamment de leur liaison à l'ADN et la surexpression de la première diminue la concentration libre de la deuxième par titration, d'où le terme séquestration (figure 3). Afin d'être opérationnel, ce mécanisme ne requiert que la surface d'interaction entre les deux protéines, et il n'est donc pas nécessaire que le répresseur lie l'ADN directement. L'interaction entre les récepteurs nucléaires et les protooncogènes précoces, jun et fos, qui relayent les effets de différentes voies de transduction des signaux intracellulaires, s'effectuerait par ce type de mécanisme [9, 19].

Arrimage. On a récemment proposé un autre mécanisme pour expliquer la répression par les récepteurs nucléaires en l'absence d'interactions directes entre le récepteur et l'ADN. La répression par "arrimage" (tethering) résulte de la liaison du récepteur à un facteur de transcription activateur qui est lui-même lié à sa séquence cible sur l'ADN. Le récepteur agit ainsi comme répresseur en bloquant la fonction de transactivation de l'activateur (figure 3). On a proposé ce mécanisme pour expliquer la répression du gène de la collagénase par les glucocorticoïdes [20, 21].

\section{La pro-opiomélanocortine (POMC)}

Les glucocorticoïdes sont synthétisés dans les surrénales en réponse à l'hormone corticotrope, l'ACTH, produite par maturation de la POMC. L'axe hypothalamo-hypo- 
physo-surrénalien est auto-réglé par des effets de rétroaction négative produits par les glucocorticoïdes plasmatiques au niveau de l'hypothalamus et de l'hypophyse. Dans l'hypophyse antérieure, l'effet de rétroaction négative des glucocorticoïdes s'exerce à un double niveau, cclui de la sécrétion de l'ACTH et celui de la transcription du gène de la POMC [22]. Ces deux effets semblent indépendants puisque l'inhibition de la sécrétion est aussi rapide que la répression de la transcription et que l'effet transcriptionnel n'influence la synthèse de l'hormone qu'à plus long terme du fait de la longue demi-vie de l'ARN messager de la POMC [23].

Un site de liaison inerte du RG. Nous avons identifié des sites de liaison du RG dans le promoteur du gène de la POMC de rat. Un de ces sites localisé à -64 pb dans le promoteur semble nécessaire à l'action des glucocorticoïdes puisque sa délétion ou sa mutation abolit la réponse hormonale [24]. Bien que ce site ne présente qu'une homologic limitée avec l'ERG positif typique, son affinité pour le RG est cependant similaire. La séquence de l'ERGn du gène POMC est comparée au consensus ERG à la figure $4 A$. Nous avons récemment montré qu'in vitro, le récepteur des glucocorticoïdes purifié à partir du foie de rat ou un fragment de celui-ci produit dans des bactéries, forme des complexes spécifiques avec l'ERGn : ces complexes contiennent trois molécules de récepteur [25]. La différence de stoechiométrie entre les complexes formés avec l'ERG consensus et l'ERGn de POMC est très évidente lorsque des sondes d'oligonucléotides synthétiques sont liées à un fragment du $\mathrm{RG}$ de 85 acides aminés qui ne contient que la région des doigts de zinc du récepteur : la figure $5 \mathrm{~A}$ montre bien que la première sonde forme des complexes monomériques et surtout dimériques, alors que l'ERGn forme en plus des complexes qui contiennent trois molécules de RG. Il semble donc que c'est une propriété intrinsèque de la séquence d'ADN et du domaine de liaison à l'ADN du RG que de former des complexes à deux ou trois sous-unités. De tels complexes n'ont jamais été décrits auparavant et ils contrastent avec la forme homodimé- rique du récepteur qui lie les $E R G$ positifs [6]. Alors que la liaison des homodimères du RG se fait du même côté de la double hélice telle qu'illustrée à la figure $4 B$, les complexes formés avec l'ERGn contiennent un homodimère qui interagit avec une face de la double hélice, comme dans le cas des ERG positifs, et une troisième molécule de récepteur qui interagit avec le sillon majeur accessible de l'autre côté de la molécule d'ADN et qui se trouve entre les séquences d'interaction des deux autres monomères du récepteur. Ainsi l'ERGn est lié de part et d'autre par ces trois molécules de RG qui ne constituent pas à proprement parler un trimère mais plutôt un homodimère et un monomère (figure 4B). Par conséquent, l'ERGn de POMC est un site de haute affinité pour le RG comme les ERG positifs mais, à la différence de ceux-ci, il lie trois molécules de récepteur plutôt que deux.

L'ERGn de POMC se comporte comme un site de liaison incrte. En effet, bien qu'il lie le RG avec une affinité élevée, ce site ne suffit pas à conférer une réponse aux glucocorticoïdes, que ce soit une réponse positive ou négative [25]. Il semble que la formation des complexes à trois molécules de RG empêche ceux-ci d'exercer la fonction transactivatrice qui est caractéristique du RG dimérique associé aux ERG positifs. Nos observations suggèrent que la conformation du récepteur au-delà du domaine de liaison à l'ADN diffère dans les deux types de complexe, et que cela affecte de façon déterminante l'activité transcriptionnelle du récepteur (figure $5 B$ ). Nos observations démontrent aussi que l'ERGn de POMC ne se comporte pas comme un répresseur direct ou un silencer. Un autre mécanisme doit donc être envisagé pour expliquer l'action répressive de ce site sur la transcription du gène de la POMC.

Mécanisme de la répression de POMC par les glucocorticoïdes. L'ERGn de la POMC étant un site de liaison inerte du $R G$, il était tout à fait plausible que la liaison du $R G$ à ce site puisse réprimer la transcription par déplacement d'un facteur positif qui lierait une séquence d'ADN chevauchant l'ERGn. $\mathrm{D}$ 'autant plus que nous avons déjà montré que le facteur de transcription COUP ("chicken ovalbumin upstream promoter ") lie cet élément in vitro [23]. Toutefois, les données expérimentales vont à l'encontre de ce modèle puisque, d'une part, l'ERGn ne confère aucune activité transcriptionnelle constitutive lorsqu'il est inséré à l'intérieur du promoteur MMTV ou en amont des promoteurs de la thymidine kinase du virus herpes ou du LTR du virus de Rovs [24] et que d'autre part, la mutation de l'ERGn dans le promoteur POMC n'affecte pas la transcription constitutive du gène [26]. Si l'on considère la position de l'ERGn dans le contexte du promoteur POMC et de ses autres éléments de régulation, il est assez étonnant de constater que des 500 paires de base requises pour l'activité promotrice du gène $\mathrm{POMC}$, la seule région qui ne semble pas contribuer à l'activité constitutive (ou de base), soit justement celle qui entoure l'ERGn. Nous devons par conséquent envisager d'autres mécanismes afin d'expliquer la répression de la transcription du gène $P O M C$ par les glucocorticoïdes.

Un mécanisme de répression par cooccupation est aussi peu probable pour les mêmes raisons que celles invoquées plus haut pour exclure un modèle de répression par compétition. Il semble donc que le modèle le plus simple pourrait impliquer un mécanisme d'interférence par lequel la liaison du RG s'interpose pour entraver l'activité du promoteur. Ainsi, le RG pourrait diminuer l'activité transcriptionnelle en se liant dans la région proximale du promoteur et ainsi perturber des interactions entre protéines liées à l'ADN en amont et en aval de l'ERGn ; par exemple, la présence d'un complexe à trois molécules de RG pourrait causer un emcombrement stérique empêchant l'interaction entre des facteurs liés en amont au promoteur et la machinerie de base de la transcription. D'autre part, la formation de ces mêmes complexes pourrait gêner la transcription en changeant ou en restreignant la conformation de l'ADN dans la région proximale du promoteur. Cela est d'autant plus envisageable qu'il a déjà été montré que le récepteur des œstrogènes induit par sa liaison une courbure de l'ADN [27]. L'hypothèse 
A

ERG

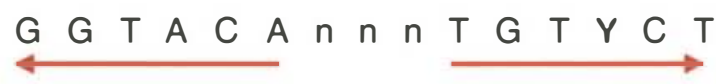

B

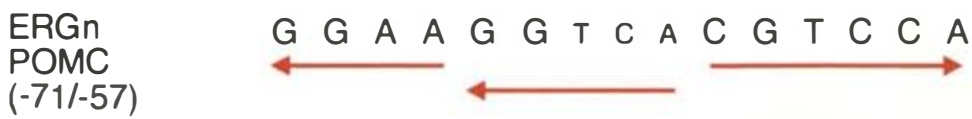

ERG

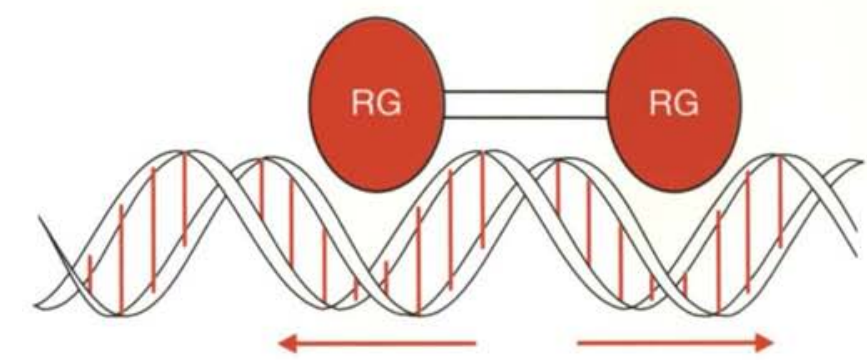

ERGn

POMC

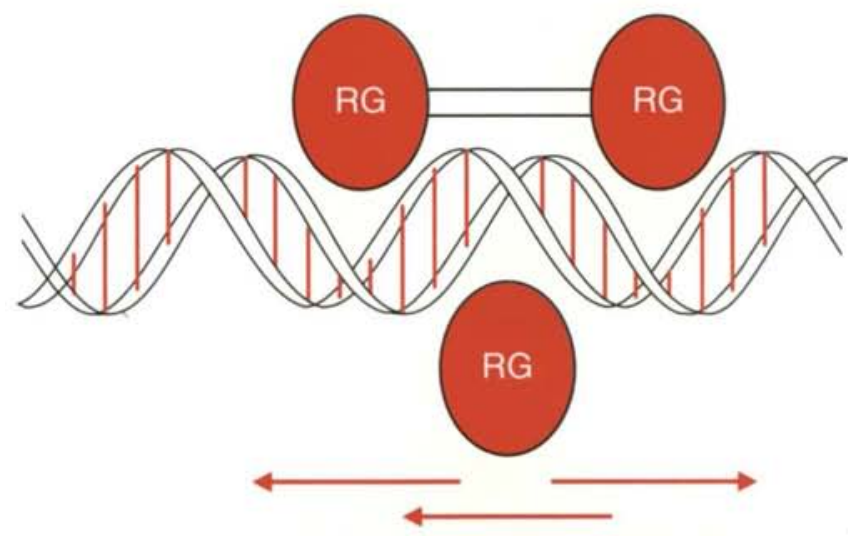

C

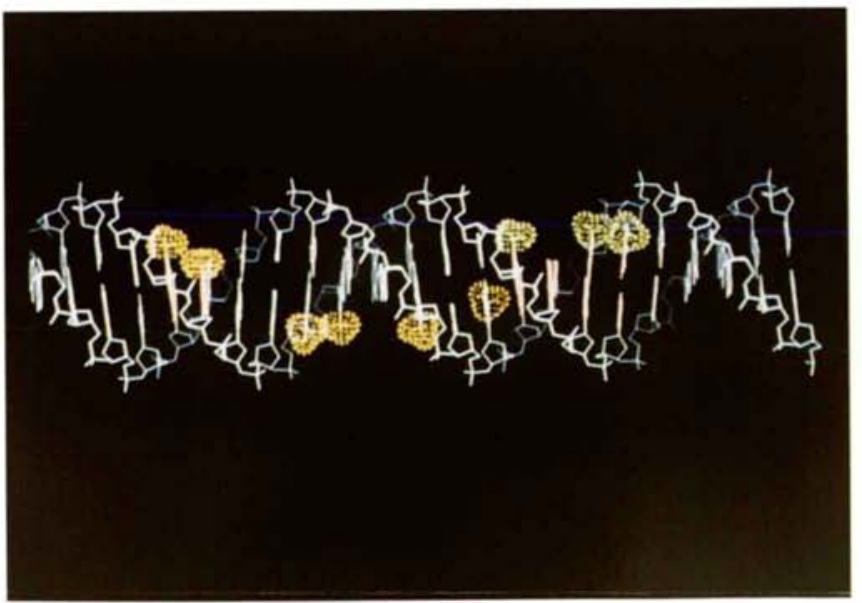

d'un effet sur la conformation de l'ADN est vraisemblable si l'on considère en plus la séquence du site de liaison de la troisième molécule du RG dans les complexes ERGn. En effet, cette séquence représente un demi-ERE, une séquence qui habituellement ne lie pas le RG [25]. Étant donné qu'un court polypeptide de 85 acides aminés peut former un complexe à trois molécules (figure 5A), il est plus vraisemblable que la liaison coopérative de cette troisième molécule dépende d'un changement de conformation du substrat ADN plutôt que d'une interaction protéine : protéine entre l'homodimère $\mathrm{RG}$ et la troisième molécule de $\mathrm{RG}$, bien que cette dernière possibilité n'ait pas été complètement exclue. Il sera intéressant de déterminer l'effet de la liaison du RG sur la conformation de l'ERG et de l'ERGn. Un effet de conformation pourrait expliquer la difficulté de conférer la réponse négative aux glucocorticoïdes par insertion de l'ERGn parce que dans ce cas, le contexte du promoteur pourrait être essentiel à la réponse transcriptionnelle négative. Cela pourrait expliquer que nous ayons pu conférer la réponse transcriptionnelle par insertion d'un fragment du pro-

Figure 4. Topologie de l'interaction du RG avec I'ERG positif consensus et l'ERGn du gène de la POMC. A) Ces séquences sont comparées et les flèches indiquent la position des hexanucléotides conservés que l'on retrouve dans les ERG positifs [3]. B) Différents modes d'interaction du RG avec I'ADN. L'interaction des homodimères RG avec I'ERG positif est semblable à celle de l'homodimère liant I'ERGn de la POMC. Une troisième molécule de RG lie cependant ce site de l'autre côté de la double hélice [25]. C) Représentation tridimensionnelle des sites d'interaction des trois sous-unités de RG avec I'ERGn de la POMC. La séquence est orientée dans le même sens que dans la partie supérieure de la figure et les bases en magenta représentent les nucléotides conservés dans I'ERGn par comparaison avec I'ERG consensus [3]. Les groupes méthyles dont la présence sur les guanines interfère avec la liaison du RG sont présentés en jaune ou en vert, et leur position indique des sites de contact intime entre le RG et I'ADN [25]. 
Figure 5. A) Liaison in vitro d'un fragment de 85 acides aminés du RG produit dans des bactéries et contenant le domaine de liaison à I'ADN (DBD) de ce récepteur. La formation d'un complexe récepteur: ADN est révélée par la méthode de retard sur gel en utilisant des sondes ERG ou ERGn. Les complexes contenant une, deux ou trois molécules d'ERG sont libellés DBD 1, DBD2 et $D B D 3$ respectivement [25]. BI La séquence de l'ADN du site de liaison du récepteur des glucocorticoiddes détermine l'effet transcriptionnel de cette interaction. Dans le cas de la liaison d'homodimère du récepteur (RG) à un élément de réponse aux glucocorticoïdes (ERG), l'effet transcriptionnel est positif, alors que la liaison de trois molécules de RG à l'ERGn du gène de la POMC réprime la transcription de ce gène.

\section{RÉFÉRENCES}

27. Nardulli AM, Shapiro DJ. Binding of the estrogen receptor DNA-binding domain to the estrogen response element induces DNA binding. Mol Cell Biol 1992; 12: 2037-42.

28. Zhang X-K, Wills $\mathrm{KN}$, Husmann $\mathrm{M}$, Hermann T, Pfahl M. Novel pathway for thyroid hormone receptor action through interaction with jun and fos oncogene activities. Mol Cell Biol 1991; 11: 6016-25.

29. Desbois C, Auber D, Legrand C, Pain B, Samarut J. A novel mechanism of action for v-ErbA : abrogation of the inactivation of transcription factor AP-1 by retinoic acid and thyroid hormone receptors. Cell 1991 ; $67: 731-40$.

30. Nicholson RC, Mader S, Nagpal S, Leik M, Rochette-Egly C, Chambon P. Negative regulation of the rat stromelysin gene promoter by retinoic acid is mediated by an API binding site. EMBOJ $1990 ; 9$ : 4443-54.

31. Yang-Yen HF, Zhang XK, Graupner G, Tzukerman M, Sakamoto B, Karin M, Pfahl M. Antagonism between retinoic acid receptors and AP-1 : implications for tumor promotion and inflammation. New Biol $1991 ; 3: 1-14$.

32. Doucas V, Spyrou G, Yaniv M. Unregulated expression of c-jun or c-fos proteins but not jun $\mathrm{D}$ inhibits oestrogen receptor activity in human breast cancer derived cells. EMBO J 1991; 10 : 2237-45.

33. Gaub M-P, Bellard M, Scheuer I, Chambon P, Sassone-Corsi P. Activation of the ovalbumin gene by the estrogen receptor involves the fos-jun complex. Cell 1990 ; 63 : 1267-76.
A

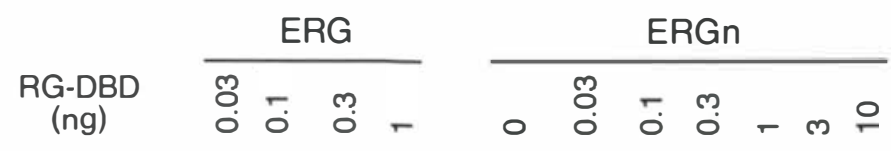

$\mathrm{DBD}_{2}-$

$\mathrm{DBD}_{1}-$
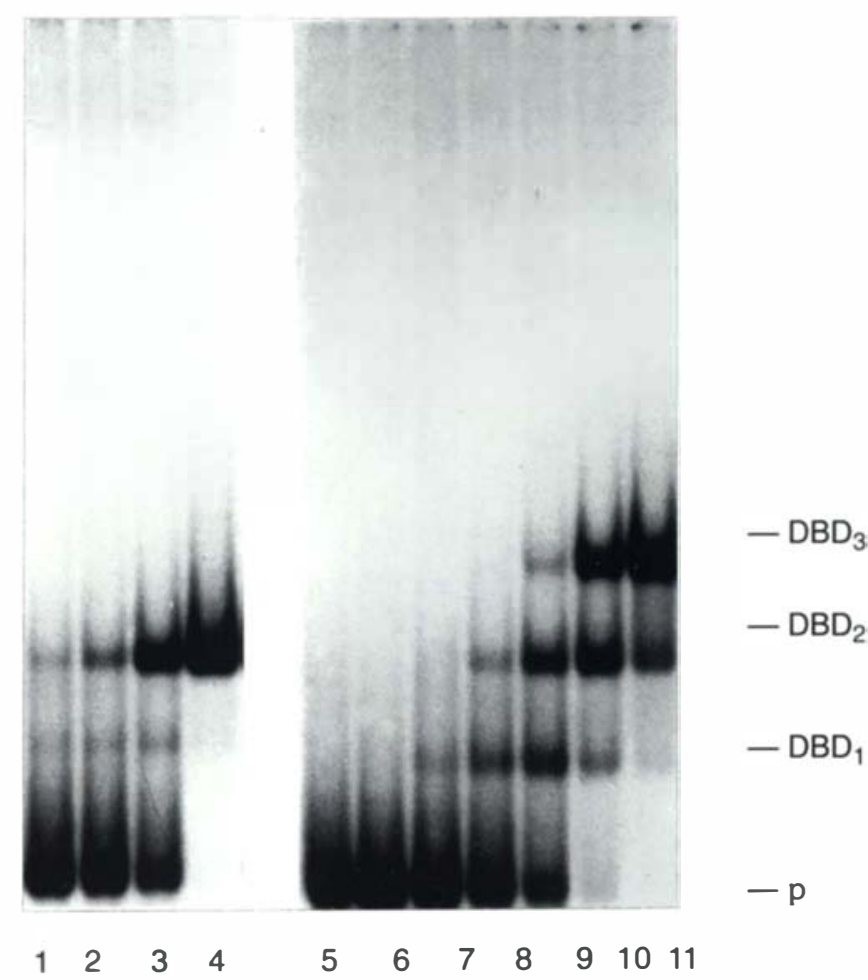

$\begin{array}{llll}1 & 2 & 3 & 4\end{array}$

$\begin{array}{llllll}6 & 7 & 8 & 9 & 10 & 11\end{array}$

B
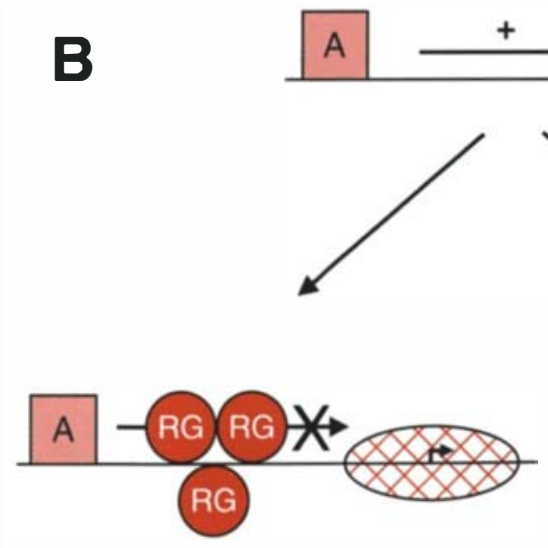

ERGn 
moteur POMC en amont d'un promoteur viral, mais qu'il n'a pas été possible de conférer cette réponse avec des oligonucléotides contenant l'ERGn [24]. Quoi qu'il en soit, ces hypothèses sur le mécanisme de la répression du gène POMC permettent des prédictions spécifiques quant à l'effet de mutations du promoteur sur la réponse hormonale, et l'approche expérimentale apportera bientôt la vérification de l'hypothèse de la répression par interférence.

\section{Perspective générale}

S'il se confirme, ce modèle de répression du gène de la POMC par interférence représentera-t-il un mécanisme général de la répression transcriptionnelle par les récepteurs nucléaires? Les données disponibles ne suggèrent pas que ce soit le cas. Il semblerait plutôt qu'un mécanisme de séquestration ou d'arrimage entre récepteurs nucléaires et facteurs d'une autre famille comme par exemple, ceux de la famille AP-1, c-jun, c-fos ou même CREB, soit utilisé dans plusieurs cas, notamment pour le gène de la collagénase $[9,19-21]$. Ces mécanismes ont aussi été invoqués pour expliquer une répression transcriptionnelle dépendante du RT [28, 29], du récepteur $\operatorname{RAR}[30,31]$, et une interaction avec le récepteur $\mathrm{RE}[32,33]$. Il n'est pas encore possible de dégager une image claire de la façon dont les interactions multiples entre différents récepteurs nucléaires et différents facteurs de la famille AP-1 [8] s'intègrent pour apporter une réponse transcriptionnelle cohérente aux multiples signaux extracellulaires (hormonaux ou autres) qui agissent sur ces voies de signalisation. De toute évidence, nous n'avons encore qu'une vision très partielle des mécanismes par lesquels les récepteurs nucléaires répriment la transcription et de la façon dont ces effets transcriptionnels intègrent les signaux multiples relayés par différentes voies de transduction, mais nous espérons que cet article aura su présenter les problèmes dans un cadre formel qui facilitera aux lecteurs l'approche de ces problèmes

\section{Summary}

Transcriptional repression : glucocorticoïds and pro-opiomelanocortin

In contrast to the wealth of information on the mechanisms responsible for activation of transcription by nuclear receptors, little is known about molecular mechanisms involved in repression of gene expression by the same nuclear receptors. Various mechanisms have been proposed and they are reviewed here, including those which require direct interaction between nuclear receptor and DNA and those which do not require such interaction but rather result from protein/protein interactions.

The gene encoding proopiomelanocortin (POMC) provides an interesting model to study repression of transcription by the glucocorticoid receptor (GR), as POMC transcription is specifically repressed by glucocorticoids in anterior pituitary corticotroph cells. We have identified an in vitro binding site for the GR within the POMC promoter and it appears to be required for glucocorticoid repression. This receptor binding site has only limited homology with positive glucocorticoid response elements (GRE). Detailed in vitro analysis of receptor-DNA interactions revealed that three molecules of GR bind this site whereas receptor homodimers were previously shown to interact with positive GREs. Mutagenesis experiments have correlated formation of these complexes with glucocorticoid repression. Current data are consistent with a model of repression by interference in which receptor binding in the proximal region of the POMC promoter interferes with the action of other transcription factors binding upstream and/or downstream of GR. This interference could be due to steric hindrance or to an effect on the local conformation of DNA. The demonstration of novel receptor-DNA complexes associated with glucocorticoid regulation of POMC suggests new mechanisms through which nuclear receptors might act to modulate gene transcription.

\section{Remerciements}

Le travail effectué par notre laboratoire n'a été possible qu'avec la contribution de nombreux collègues au cours des ans : ce sont les auteurs des publications originales cités dans cet article de synthèse. Nous remercions aussi Lise Laroche pour son excellent travail de secrétariat. Ce travail a été financé par des subventions du Conseil de recherches médicales du Canada et de l'Institut national du cancer du Canada. 